\title{
Solution Structure of a Prion Protein Aptamer Analogue ${ }^{\dagger}$
}

\author{
Špela Medic, ${ }^{a}$ Peter Podbevšek, ${ }^{\text {a,b }}$ and Janez Plavec ${ }^{a, b, c, *}$

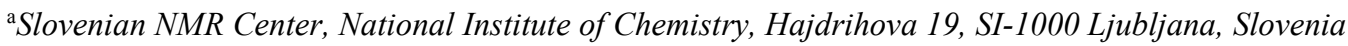 \\ ${ }^{\mathrm{b}}$ EN-FIST Centre of Excellence, Trg Osvobodilne fronte 13, SI-1000 Ljubljana, Slovenia \\ ${ }^{c}$ Faculty of Chemistry and Chemical Technology, University of Ljubljana, Aškerčeva 5, SI-1000 Ljubljana, Slovenia
}

RECEIVED FEBRUARY 7, 2014; REVISED APRIL 10, 2014; ACCEPTED MAY 9, 2014

\begin{abstract}
It has previously been shown that $\mathrm{r}(\mathrm{GGA})_{4}$ folds into a G-quadruplex structure, which binds to the normal cellular form of the prion protein $\left(\mathrm{PrP}^{\mathrm{C}}\right)$ with high affinity. The current study utilizes $\mathrm{CD}$ and NMR spectroscopy to show that a dimeric parallel G-quadruplex structure is formed by $\mathrm{r}(\mathrm{GGA})_{2}$ in a $\mathrm{KCl}$ solution. Each $\mathrm{r}\left[(\mathrm{GGA})_{2}\right]_{2}$ G-quadruplex unit exhibits two G-quartets, one of which is hydrogen bonded to two additional adenines forming a hexade. Through stacking of hexade planes, two G-quadruplex units interact with each other and form a symmetric dimer, $r\left[(\mathrm{GGA})_{2}\right]_{4}$. The topology of $\mathrm{r}\left[(\mathrm{GGA})_{2}\right]_{4}$ is in agreement with the fold of $\mathrm{r}\left[(\mathrm{GGA})_{4}\right]_{2}$, however, subtle differences are found in the region responsible for $\operatorname{PrP}^{\mathrm{C}}$ binding.
\end{abstract}

Keywords: G-quadruplex, RNA, aptamer, NMR, GGA repeat, prion protein

\section{INTRODUCTION}

Guanine rich DNA and RNA oligonucleotides are capable of folding into four-stranded structures called G-quadruplexes. ${ }^{1-5}$ The building blocks of these noncanonical structures are G-quartets, stacked planar arrangements of four guanines. G-quadruplex formation is strongly dependent on the presence of certain mono- or divalent cations. Different strand directionality, molecularity and loop conformations give rise to tremendous structural polymorphism, which is largely determined by the oligonucleotides primary structure. Consequently, small variations in the sequence of oligonucleotides can result in a large pool of structurally diverse G-quadruplexes. This plethora of structures can be exploited for the selection of G-quadruplex based aptamers with highly specific target recognition and binding. ${ }^{6,7}$ Previously, DNA and RNA oligonucleotides capable of folding into G-quadruplex structures were found to specifically interact with the prion protein. ${ }^{8,9}$

Triplet $(\mathrm{GGA})_{\mathrm{n}}$ repeats can be found throughout the human genome. ${ }^{10,11}$ It has been shown previously that such repeats are capable of forming fold-back hairpins stabilized by G:G and A:A base pairs. ${ }^{12}$ Studies suggested that oligonucleotides with GGA repeats form four-stranded structures with G-quartets as constitutive units. ${ }^{13}$ A 12-nt RNA oligonucleotide $\mathrm{r}(\mathrm{GGA})_{4}$ was identified as an aptamer for binding to the $\alpha$-helix rich cellular form of the prion protein $\left(\mathrm{PrP}^{\mathrm{C}}\right) \cdot{ }^{14}$ Subsequent NMR studies showed that $\mathrm{r}(\mathrm{GGA})_{4}$ folds into an intramolecular parallel G-quadruplex. ${ }^{15}$ In the $\mathrm{r}(\mathrm{GGA})_{4}$ G-quadruplex structure four guanine residues constitute a classical G-quartet, while the remaining four guanines form a hexade with two adenines on the sides. Two G-quadruplex units dimerize through stacking interactions between two hexade planes. The outer G-quartets are responsible for the interaction with $\mathrm{PrP}^{\mathrm{C}}{ }^{16}$ Each G-quadruplex unit binds to a portion of the N-terminal half of $\operatorname{PrP}^{\mathrm{C}}$, resulting in tight binding in the nanomolar range. It is speculated that such RNA aptamer, which stabilizes $\operatorname{PrP}^{\mathrm{C}}$, is expected to block the conversion into the $\beta$-sheet rich abnormal form $\mathrm{PrP}^{\mathrm{Sc}}$ and thereby prevent prion disease development. ${ }^{17}$

Herein we explore the possibility of the formation of a G-quadruplex structure by a shorter RNA oligonucleotide $\mathrm{r}(\mathrm{GGA})_{2}$. CD and NMR spectroscopy reveal that a G-quadruplex is readily formed by $r(\mathrm{GGA})_{2}$ in a $\mathrm{KCl}$ solution. Furthermore, two G-quadruplex units dimerize in a similar fashion as observed for $\mathrm{r}(\mathrm{GGA})_{4}$. However, subtle structural differences are found in the region responsible for interaction with the prion protein, which contribute new insights into binding of the RNA aptamer to $\operatorname{PrP}^{\mathrm{C}}$.

\footnotetext{
$\dagger$ Dedicated to Dr. Mirjana Eckert-Maksić on the occasion of her $70^{\text {th }}$ birthday.

* Author to whom correspondence should be addressed. (E-mail: janez.plavec@ki.si)
} 


\section{EXPERIMENTAL}

\section{Sample Preparation}

Uniformly isotopically ${ }^{13} \mathrm{C} /{ }^{15} \mathrm{~N}$ labeled and unlabeled RNA oligonucleotides $\mathrm{r}(\mathrm{GGA})_{2}$ were synthesized by in vitro enzymatic transcription with T7 RNA polymerase following standard protocols. ${ }^{18}$ Oligonucleotides were purified on denaturing PAGE. Ultrafiltration was used to remove salts and buffer from sample prior to NMR experiments.

NMR samples were prepared by dissolving the RNA in $\mathrm{H}_{2} \mathrm{O}$ containing $10 \%{ }^{2} \mathrm{H}_{2} \mathrm{O}$. $\mathrm{KCl}$ and EDTA were added to final concentrations of $100 \mathrm{mM}$ and 2 $\mathrm{mM}$, respectively. Samples were annealed at $95^{\circ} \mathrm{C}$ for 5 minutes followed by snap-cooling on ice for 30 minutes to ensure proper folding of the RNA. $\mathrm{pH}$ of the samples was adjusted to 6.2 with potassium phosphate buffer. The sample in ${ }^{2} \mathrm{H}_{2} \mathrm{O}$ was prepared by lyophilization of the RNA and resuspension in $100 \%{ }^{2} \mathrm{H}_{2} \mathrm{O}$. Strand concentration of the ${ }^{13} \mathrm{C} /{ }^{15} \mathrm{~N}$ double-labeled oligonucleotide was $0.5 \mathrm{mM}$ per strand and $0.3 \mathrm{mM}$ per strand for the unlabeled oligonucleotide. Concentration was determined spectrophotometrically at $260 \mathrm{~nm}$ using a Varian CARY-100 BIO Spectrophotometer.

\section{Circular dichroism Spectroscopy}

Circular dichroism (CD) spectra were recorded on an Applied Photophysics Chirascan CD spectrometer at 25 ${ }^{\circ} \mathrm{C}$ using a $1.0 \mathrm{~mm}$ path length quartz cell. The wavelength range was from 200 to $320 \mathrm{~nm}$. CD samples were prepared at $50 \mu \mathrm{M}$ oligonucleotide concentration in 100 $\mathrm{mM} \mathrm{KCl}$ and $2 \mathrm{mM}$ EDTA solution.

\section{NMR Spectroscopy}

NMR spectra were collected on Agilent VNMRS 800, 600 and $300 \mathrm{MHz}$ NMR spectrometers at $25^{\circ} \mathrm{C}$. ${ }^{1} \mathrm{H}$ 1D NMR spectra were acquired using DPFGSE water suppression sequence. The translation diffusion coefficient was determined using pulse field gradient stimulated echo pulse sequence. NOESY spectra were acquired with mixing times $\left(\tau_{\mathrm{m}}\right)$ of 100, 125, 150, 175, 200, 225 and $250 \mathrm{~ms}$. Resonances were assigned by analyzing NOESY, HSQC, HCN, HCN-TOCSY-CH and HCCH TOCSY spectra. Spectra were processed with VNMRJ (Agilet Technologies) and NMRPipe ${ }^{19}$ software packages. Signal integration with Gaussian fit procedure was done with Sparky software (UCSF). NOE distance restraints for exchangeable protons were classified as strong (1.8-3.6 $\AA$ ), medium $(2.6-5.0 \AA)$ and weak $(3.5-6.5 \AA)$.

\section{Structure Calculations}

Molecular modeling was performed using AMBER 11 software with the parmbsc 0 force field ${ }^{20,21}$ starting from an initial extended structure. A series of 100 restrained simulated annealing (SA) simulations using the Born implicit solvation model was carried out. For the first 25 ps molecules were held at a constant temperature of $1000 \mathrm{~K}$. The molecules were then cooled to $300 \mathrm{~K}$ in the next $25 \mathrm{ps}$, after which the temperature was scaled down to $100 \mathrm{~K}$ in $25 \mathrm{ps}$ and reduced to $0 \mathrm{~K}$ in the last $25 \mathrm{ps}$. Force constants were $20 \mathrm{kcal} \mathrm{mol}^{-1} \AA^{-2}$ for NOE distances, $200 \mathrm{kcal} \mathrm{mol}^{-1} \mathrm{rad}^{-2}$ for torsion angle and 400 kcal mol ${ }^{-1} \AA^{-2}$ for G-quartet base planarity restraints. The cutoff for non-bonded interactions was $20 \AA$ and SHAKE algorithm for hydrogen atoms had a tolerance of $0.0005 \AA .10$ of the lowest energy structures were selected and minimized with a maximum of 10000 steps of conjugate gradient energy minimization.

\section{RESULTS AND DISCUSSION}

Ample quantities of the $\mathrm{r}(\mathrm{GGA})_{2}$ for NMR studies were prepared with in vitro RNA synthesis utilizing T7 polymerase. 1D ${ }^{1} \mathrm{H}$ spectrum of $\mathrm{r}(\mathrm{GGA})_{2}$ reveals that a single species is formed in a $100 \mathrm{mM} \mathrm{KCl}$ solution (Figure 1). Four well resolved resonances in the imino region of the spectrum ( $\delta$ 11.0-11.5 ppm) correspond to hydrogen bonds in Hoogsteen geometry and indicate the presence of a G-quadruplex structure. The number of observable imino signals suggests the formation of a symmetric bimolecular G-quadruplex with two G-quartets.

CD spectroscopy was utilized in order to determine the strand directionality within the G-quadruplex. A positive peak at $260 \mathrm{~nm}$ and a negative peak at 240 $\mathrm{nm}$ are indicative of a parallel G-quadruplex structure (Figure 2). Interestingly, parallel orientation of the four strands in the G-quadruplex adopted by $\mathrm{r}(\mathrm{GGA})_{2}$ is reminiscent of the topology of related $\mathrm{r}(\mathrm{GGA})_{4}$ G-quadruplex.

Relevant NMR resonances were assigned with standard 2D NOESY, COSY and TOCSY based approaches. Additionally, 2D HSQC, 3D NOESY-HSQC, $\mathrm{HCN}$ and $\mathrm{HCCH}-\mathrm{COSY}$ spectra were acquired with ${ }^{13} \mathrm{C} /{ }^{15} \mathrm{~N}$ double labeled $\mathrm{r}(\mathrm{GGA})_{2}$. The sequential walk in aromatic-anomeric region of NOESY spectra could be observed throughout the $\mathrm{r}(\mathrm{GGA})_{2}$ sequence (Figure 3). It is noteworthy, however, that internucleotide $\mathrm{G} 2 \mathrm{H} 1{ }^{\prime}-\mathrm{A} 3 \mathrm{H} 8$ and $\mathrm{A} 3 \mathrm{H} 11^{\prime}-\mathrm{G} 4 \mathrm{H} 8$ cross-peaks are very weak. Also, correlations between $\mathrm{H} 8$ and N9 in A3

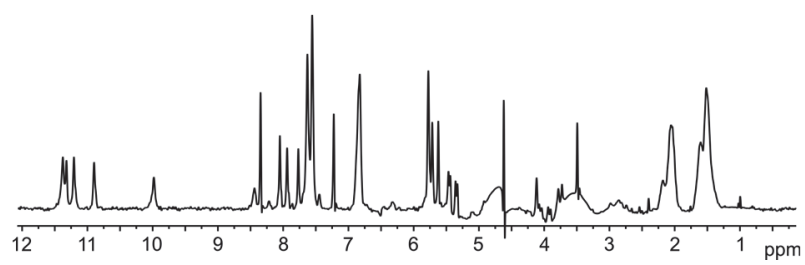

Figure 1. 1D ${ }^{1} \mathrm{H}$ NMR spectrum of $\mathrm{r}(\mathrm{GGA})_{2}$ in a $100 \mathrm{mM} \mathrm{KCl}$ solution. 


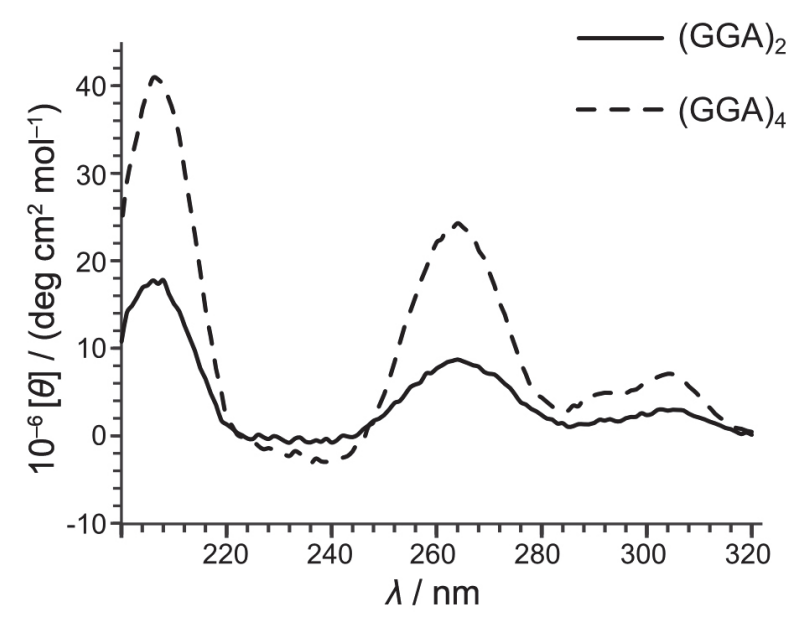

Figure 2. CD spectra of $\mathrm{r}(\mathrm{GGA})_{2}$ and $\mathrm{r}(\mathrm{GGA})_{4}$ in a $100 \mathrm{mM}$ $\mathrm{KCl}$ aqueous solution.

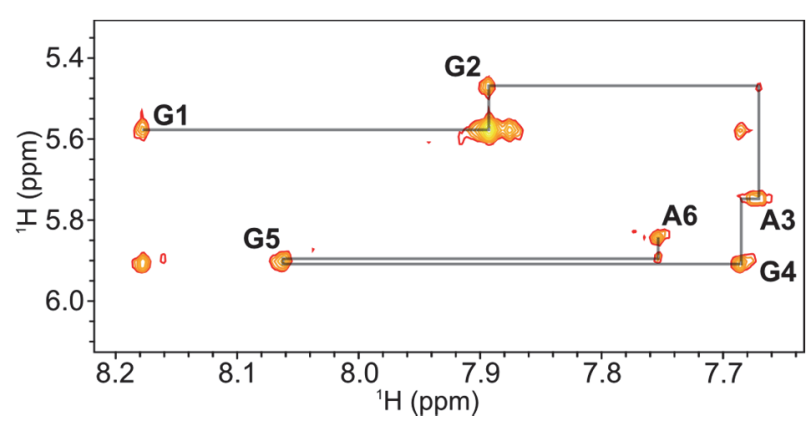

Figure 3. Aromatic-anomeric region of a NOESY spectrum $\left(\tau_{\mathrm{m}}=250 \mathrm{~ms}\right)$ with lines showing the sequential walk.

and between H1' and N9 in G4 could not be observed in HCN spectra (data not shown). This suggests considerable deviations of glycosidic torsion angles from A-type geometry in the vicinity of the $\mathrm{A} 3$ residue.

It was previously shown that a related oligonucleotide sequence can form a structure where two G-quadruplex units dimerize through the stacking of G/A hexade planes. $^{15}$ To explore this possibility hydrodynamic properties of the G-quadruplex formed by $\mathrm{r}(\mathrm{GGA})_{2}$ were assessed with the use of a ${ }^{1} \mathrm{H}-{ }^{13} \mathrm{C}$ HSQC-DOSY diffusion NMR experiment. The translational diffusion coefficient of $1.67 \times 10^{-6} \mathrm{~cm}^{2} \mathrm{~s}^{-1}$ suggests the formation of a dimeric G-quadruplex unit $\mathrm{r}\left[(\mathrm{GGA})_{2}\right]_{4}$. In comparison, the predicted diffusion coefficients for monomeric and dimeric units of the G-quadruplex formed by $\mathrm{r}(\mathrm{GGA})_{4}$ are $1.85 \times 10^{-6} \mathrm{~cm}^{2} \mathrm{~s}^{-1}$ and $1.59 \times 10^{-6} \mathrm{~cm}^{2} \mathrm{~s}^{-1}$, respectively.

Interestingly, deuterium exchange experiments showed that G1 and G4 imino and G1 amino proton resonances remained protected from exchange for weeks after the substitution of $\mathrm{H}_{2} \mathrm{O}$ for ${ }^{2} \mathrm{H}_{2} \mathrm{O}$ as solvent. This suggests an extremely tightly packed structure at the interface of the two G-quadruplex units. This is in agreement with findings that G1, G4, G7 and G10 imino resonances of $\mathrm{r}\left[(\mathrm{GGA})_{4}\right]_{2}$ also remain observable for at least a day after the change of solvent. ${ }^{15}$

Simulated annealing was used to calculate a 3D structure based on the predicted topology and NMR distance restraints. The structure consists of two $\mathrm{r}(\mathrm{GGA})_{2}$ oligonucleotide chains, designated $\mathbf{a}$ and $\mathbf{b}$, which form one bimolecular G-quadruplex unit, and two additional chains, $\mathbf{c}$ and $\mathbf{d}$, which constitute the symmetric G-quadruplex unit (Figure 4). Each nucleus therefore exhibits a 4-fold symmetry. According to intensities of internucleotide H8-H1' NOESY cross-peaks, the $\chi$ angles of all nucleotides were assigned to anti conformation. Due to the absence of observable H1'-H2' TOCSY cross-peaks all sugar pucker conformations were restrained to $\mathrm{C} 3$ '-endo. Several NOE contacts, which could not be satisfied within a single G-quadruplex unit, due to large distances, have been assigned to nuclei between the two G-quadruplex units (Figure 4). A standard simulated annealing protocol was used to calculate a set of 100 structures of which 10 lowest energy structures were selected for further refinement. Heavy atom RMSD value of the 10 lowest energy G-quadruplex structures is $1.20 \AA$. Guanines involved in G-quartets give an RMSD of $0.71 \AA$. The structure of $A 3$, which is hydrogen bonded to G1, is also well defined with a RMDS value of $0.64 \AA$, while terminal A6 residue exhibits a relatively large RMSD of $2.51 \AA$.

The symmetric structure consists of two G-quadruplexes, each comprised of two G-quartets (Figure 5a). The inner G-quartets, comprised of residues G1 and G4, are located at the interface of the two G-quadruplex units. The remaining G2 and G5 residues form the outer G-quartets. Residue A3, which is connecting two G-tracts, does not form a classic propeller-type loop. It is hydrogen bonded to G1 and therefore positioned in the plane of the inner G-quartet. However, A3 nucleobase is not parallel to the G-quartet plane, but set at an approximately $45^{\circ}$ angle. Interestingly, the nucleobases of two adjacent $\mathrm{A} 3$ residues belonging to different monomeric G-quadruplex units (a.A3-c.A3 and b.A3-d.A3) are parallel to each other. 3' terminal A6 residues are positioned above the outer G-quartets, however, they are highly mobile and therefore not well stacked on the purine moieties of G2 or G5.

\section{CONCLUSION}

Molecularity plays an important role in G-quadruplex formation. Monomolecular G-quadruplexes are expected to fold instantly in solution. On the other hand, bimolecular and especially teramolecular G-quadruplex often fold slowly and the rate of folding is highly concentration dependent. In this study we describe the 


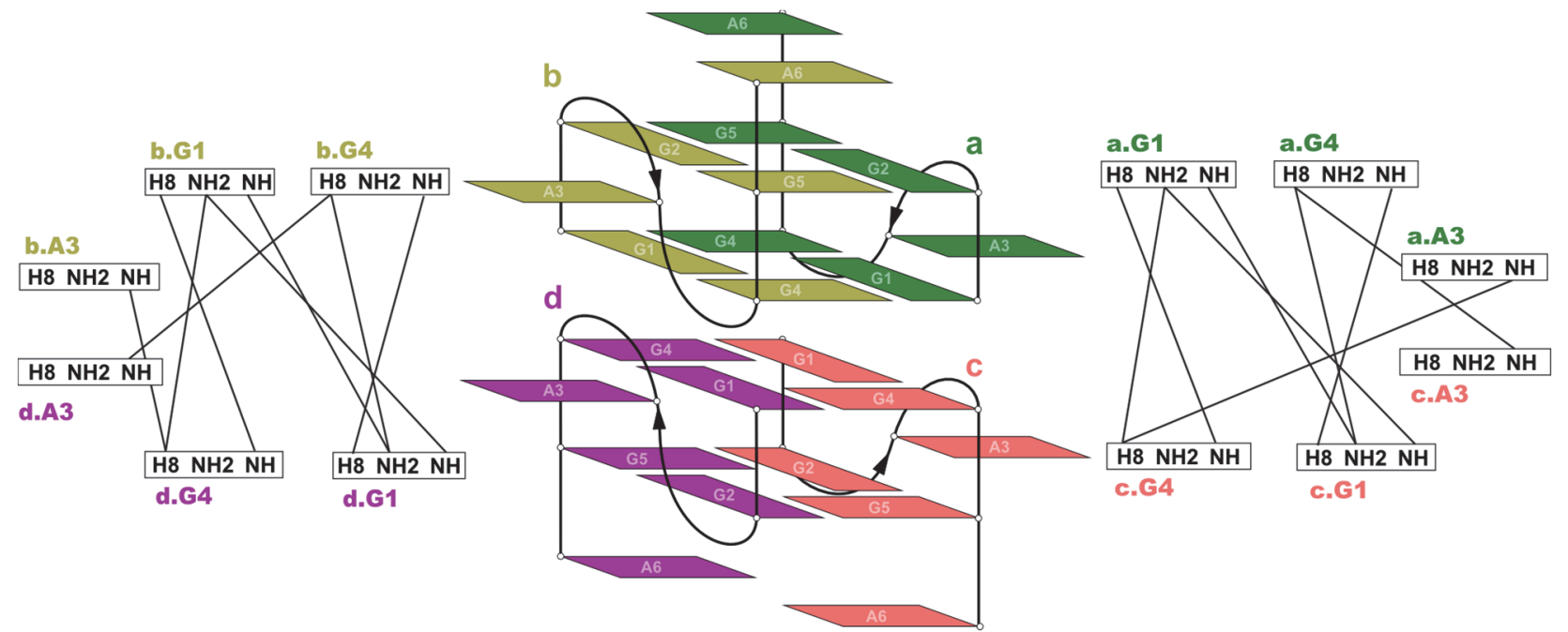

Figure 4. Topology of the $\mathrm{r}\left[(\mathrm{GGA})_{2}\right]_{4}$ dimeric G-quadruplex with a schematic presentation of NOE contacts at the interface of two G-quadruplex units.

structure of a bimolecular G-quadruplex, which not only folds readily in solution, but also dimerizes in a similar fashion as its monomolecular counterpart.

Although the topologies of $\mathrm{r}\left[(\mathrm{GGA})_{2}\right]_{4}$ and $\mathrm{r}\left[(\mathrm{GGA})_{4}\right]_{2}$ are largely the same, subtle but significant differences in the structural features of the two G-quadruplexes have been observed. While the structure of guanine residues comprising the core of G-quadruplexes and the way the two monomeric units interact with each other are very similar, there are noticeable discrepancies in the positions of adenine residues (Figure 5). A3 and A9 nucleobases in $r\left[(\mathrm{GGA})_{4}\right]_{2}$ are an integral part of the mixed G/A hexade and completely parallel to the G-quartet plane, while $\mathrm{A} 3$ bases in $\mathrm{r}\left[(\mathrm{GGA})_{2}\right]_{4}$ are oriented at an approximately $45^{\circ}$ angle to the G-quartet plane, however, still hydrogen bonded to G1 with a single A3H62-N3G1 bond. Interestingly, this orientation of A3 residues apparently does not results in a less compact structure, as G1 and G4 exchangeable proton resonances persist for weeks in deuterated water.

More significantly, is has been shown that the interaction between $\operatorname{PrP}^{\mathrm{C}}$ and $\mathrm{r}\left[(\mathrm{GGA})_{4}\right]_{2}$ is mediated through both outer G-quartets of the dimerized G-quadruplexes. Two (one per G-quadruplex unit) terminal A12 residues in $r\left[(G G A)_{4}\right]_{2}$ are stacked on the outer G-quartets. However upon $\operatorname{PrP}^{\mathrm{C}}$ binding A12 rotates to the side of the G-quadruplex structure, leaving the outer G-quartets accessible for interaction with the unstructured (a)

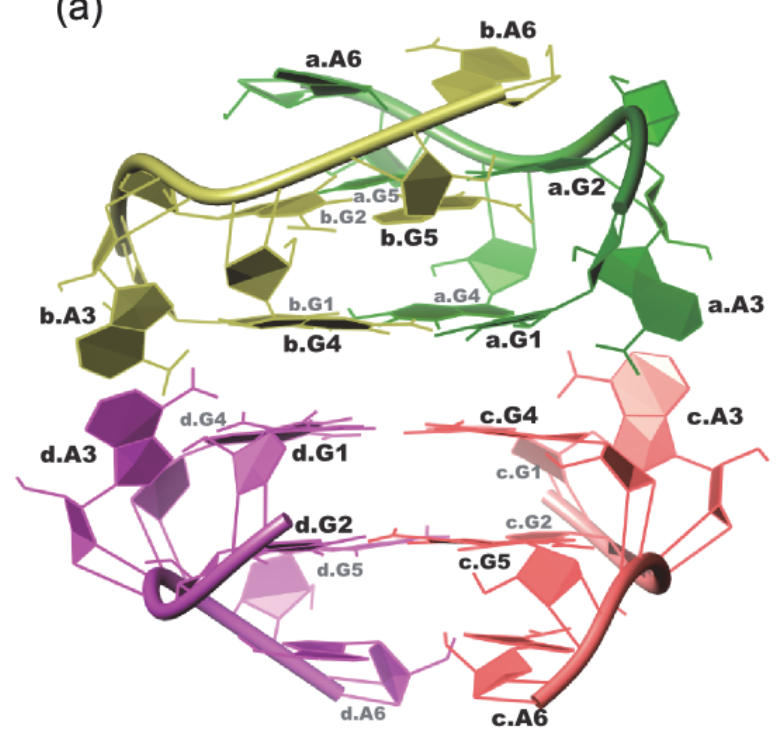

(b)

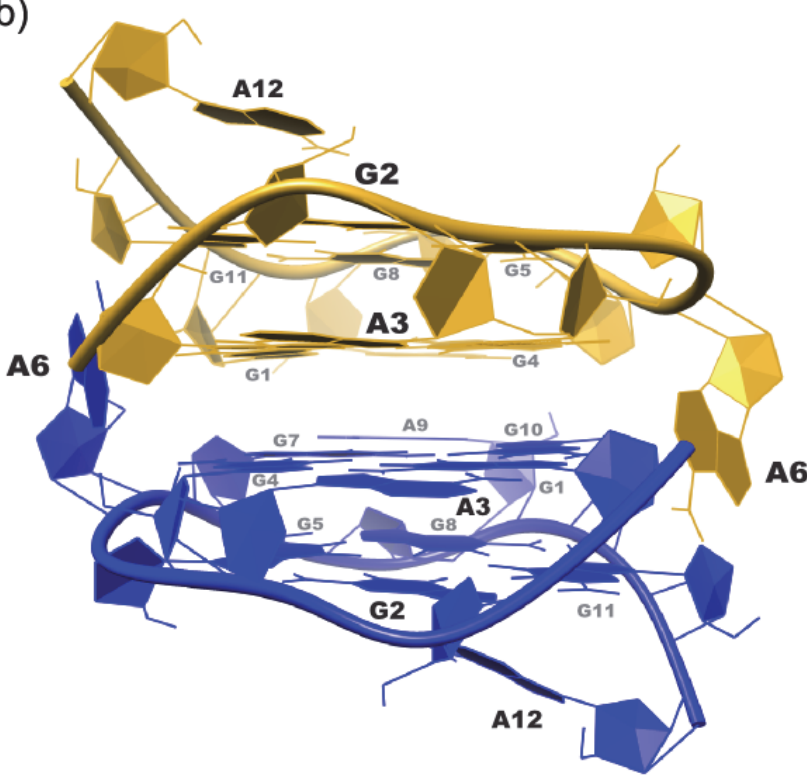

Figure 5. Comparison of representative structures of dimeric G-quadruplexes formed by $r\left[(\mathrm{GGA})_{2}\right]_{4}$ (a) and $r\left[(\mathrm{GGA})_{4}\right]_{2}(\mathrm{~b})$. Individual strands are represented with different colors. 
N-terminal region of $\operatorname{PrP}^{\mathrm{C}} \cdot{ }^{15,16}$ On the other hand, four terminal A6 residues (two per G-quadruplex unit) of $\mathrm{r}\left[(\mathrm{GGA})_{2}\right]_{4}$, while still somewhat flexible, are positioned above the outer G-quartets and most likely sterically hinder possible external interactions. While A6 residues could rotate to the sides of the G-quadruplex structure, a barrier of repositioning of four instead of two nucleotides would have to be overcome upon $\operatorname{PrP}^{\mathrm{C}}$ binding. We believe this would result in an increase in the binding constant and make $r\left[(\mathrm{GGA})_{4}\right]_{2}$ a less effective anti-prion protein aptamer.

Acknowledgements. This work was supported by the Slovenian Research Agency (ARRS, grants P1-0242 and J1-4020), Bio-NMR FP7 project (contract 261863) and European Cooperation in Science and Technology (COST MP0802).

\section{REFERENCES}

1. S. Neidle and S. Balasubramanian, Quadruplex nucleic acids, Royal Society of Chemistry, Cambridge, 2006.

2. W. Fritzsche and L. Spindler, Guanine Quartets: Structure and Application, Royal Society of Chemistry, Cambridge, 2013.

3. J. T. Davis, Angew. Chem. Int. Ed. 43 (2004) 668-698.

4. A. T. Phan, V. Kuryavyi and D. J. Patel, Curr. Opin. Struc. Biol. 16 (2006) 288-298.

5. S. Burge, G. N. Parkinson, P. Hazel, A. K. Todd and S. Neidle, Nucleic Acids Res. 34 (2006) 5402-5415.
6. B. Gatto, M. Palumbo, and C. Sissi, Curr. Med. Chem. 16 (2009) 1248-1265.

7. G. W. Collie and G. N. Parkinson, Chem. Soc. Rev. 40 (2011) 5867-5892.

8. S. Weiss, D. Proske, M. Neumann, M. H. Groschup, H. A. Kretzschmar, M. Famulok, and E. L. Winnacker, J. Virol. 71 (1997) 8790-8797.

9. P. Cavaliere, B. Pagano, V. Granata, S. Prigent, H. Rezaei, C. Giancola, and A. Zagari, Nucleic Acids Res. 41 (2013) 327-339.

10. G. Tóth, Z. Gáspári, and J. Jurka, Genome Res. 10 (2000) 967-981.

11. P. Kozlowski, M. de Mezer, and W. J. Krzyzosiak, Nucleic Acids Res. 38 (2010) 4027-4039.

12. D. Huertas and F. Azorín, Biochemistry 35 (1996) 13125-13135.

13. K. Usdin, Nucleic Acids Res. 26 (1998) 4078-4085.

14. K. Murakami, F. Nishikawa, K. Noda, T. Yokoyama, and S. Nishikawa, Prion 2 (2008) 73-80.

15. T. Mashima, A. Matsugami, F. Nishikawa, S. Nishikawa, and M. Katahira, Nucleic Acids Res. 37 (2009) 6249-6258.

16. T. Mashima, F. Nishikawa, Y. O. Kamatari, H. Fujiwara, M. Saimura, T. Nagata, T. Kodaki, S. Nishikawa, K. Kuwata, and M. Katahira, Nucleic Acids Res. 41 (2013) 1355-1362.

17. D. Proske, S. Gilch, F. Wopfner, H. M. Schätzl, E.-L. Winnacker, and M. Famulok, ChemBioChem 3 (2002) 717-725.

18. J. F. Milligan and O. C. Uhlenbeck, Meth. Enzymol. 180 (1989) 51-62.

19. F. Delaglio, S. Grzesiek, G. W. Vuister, G. Zhu, J. Pfeifer, and A. Bax, J. Biomol. NMR 6 (1995) 277-293.

20. W. D. Cornell, P. Cieplak, C. I. Bayly, I. R. Gould, K. M. Merz, D. M. Ferguson, D. C. Spellmeyer, T. Fox, J. W. Caldwell, and P. A. Kollman, J. Am. Chem. Soc. 117 (1995) 5179-5197.

21. A. Perez, I. Marchan, D. Svozil, J. Sponer, T. Cheatham, C. Laughton, and M. Orozco, Biophys. J. 92 (2007) 3817-3829. 